https://helda.helsinki.fi

\title{
An efficient auxin-inducible degron system with low basal degradation in human cells
}

\section{$\mathrm{Li}$, Shiqian}

2019-09

Li , S , Prasanna , X , Salo , V T , Vattulainen , I \& Ikonen , E 2019 , ' An efficient auxin-inducible degron system with low basal degradation in human cells ', Nature methods , vol. 16 , no. 9 , pp. 866-+ . https://doi.org/10.1038/s41592-019-0512-x

http://hdl.handle.net/10138/319568

https://doi.org/10.1038/s41592-019-0512-x

unspecified

publishedVersion

Downloaded from Helda, University of Helsinki institutional repository.

This is an electronic reprint of the original article.

This reprint may differ from the original in pagination and typographic detail.

Please cite the original version. 


\title{
An efficient auxin-inducible degron system with low basal degradation in human cells
}

\author{
Shiqian Li ${ }^{1,2}$, Xavier Prasanna ${ }^{3,5}$, Veijo T. Salo $\rrbracket^{1,2,5}$, Ilpo Vattulainen $\mathbb{1}^{3,4}$ and Elina Ikonen $\left(\mathbb{1}^{1,2 \star}\right.$
}

\begin{abstract}
Auxin-inducible degron technology allows rapid and controlled protein depletion. However, basal degradation without auxin and inefficient auxin-inducible depletion have limited its utility. We have identified a potent auxin-inducible degron system composed of auxin receptor F-box protein AtAFB2 and short degron minilAA7. The system showed minimal basal degradation and enabled rapid auxin-inducible depletion of endogenous human transmembrane, cytoplasmic and nuclear proteins in $1 \mathrm{~h}$ with robust functional phenotypes.
\end{abstract}

Loss-of-function methods for reducing target protein levels target DNA, RNA or protein ${ }^{1}$. Auxin-inducible degron (AID) technology allows rapid targeted protein depletion with the smallmolecule auxin ${ }^{2}$. To apply AID, an auxin-inducible destabilizing domain, or 'degron', is fused to the target protein. In addition, an auxin receptor F-box protein TIR1/AFB is exogenously expressed, forming a chimeric SKP1-CUL1-F-Box (SCF) ubiquitin E3 ligase with endogenous components in eukaryotic cells. The chimeric E3 ligase recruits the degron in an auxin-dependent manner ${ }^{3}$. Addition of indole-3-acetic acid (IAA) or other small molecules of the auxin class to the culture medium thus induces the polyubiquitination and rapid proteasomal degradation of the degron-fused protein (Fig. 1a). This approach has been used as a powerful tool to acutely deplete target proteins in studying their functions $s^{4-7}$. However, current AID systems can severely degrade target proteins before IAA addition (known as 'basal degradation') and suffer from inefficient auxin-inducible depletion in a context- and target-specific manner ${ }^{2,8-10}$. These pitfalls have substantially limited the applications of AID technology. An AID system with both minimal basal degradation and rapid auxin-inducible depletion would be desirable.

In current AID systems, Oryza sativa TIR1 (OsTIR1) is the most commonly used auxin receptor F-box protein in combination with different degrons deriving from Arabidopsis thaliana IAA17 (AtIAA17) $2,4,5,7-14$. We initially aimed to acutely deplete endogenous human seipin, a transmembrane protein involved in lipid droplet (LD) biogenesis. However, seipin tagged with a degron termed miniAID (composed of AtIAA17 amino acid (aa) 68-132) was severely degraded in cells expressing OsTIR1 without IAA addition. Consequently, cells exhibited defective LD biogenesis already before IAA addition, resembling a seipin knockout phenotype ${ }^{15}$ (Supplementary Fig. 1a-c).

To search for an improved AID system, we established a pipeline in human A431 cells for screening various auxin receptor F-box proteins and degrons, through co-integration into the adenoassociated virus integration site 1 (AAVS1) safe harbor locus ${ }^{16}$ (Fig. 1b and Supplementary Note 1). We first compared OsTIR1 to five other auxin receptor F-box proteins, using miniAID as the degron. A. thaliana AFB2 (AtAFB2) was identified as the best hit: compared to OsTIR1, it displayed minimal basal depletion with over five-fold higher target protein level at $0 \mathrm{~h}$ IAA and similar auxin-inducible depletion at $16 \mathrm{~h}$ IAA treatment (Fig. 1c and Supplementary Fig. 2a-e). We speculate that AtAFB2 was superior to the other proteins tested probably because of its better thermostability at $37^{\circ} \mathrm{C}$, analogously to OsTIR1 (ref. ${ }^{2}$ ). However, auxininducible depletion with AtAFB2 at $1 \mathrm{~h}$ IAA was inefficient. We next compared miniAID to 14 other degrons, in combination with either AtAFB2 or OsTIR1. All degrons showed severe basal degradation at $0 \mathrm{~h}$ IAA with OsTIR1 but not with AtAFB2 (Supplementary Fig. 3a-c). A degron composed of AtIAA7 amino acids 37-104 (hereafter denoted 'miniIAA7') was identified as an optimal degron in combination with AtAFB2. It dramatically improved auxin-inducible depletion with over three-fold more efficient protein reduction compared to miniAID at $1 \mathrm{~h}$ IAA (Fig. $1 \mathrm{~d}$ and Supplementary Fig. 3a-c). Thus, the AtAFB2-miniIAA7 system showed both minimal basal degradation and rapid auxin-inducible depletion.

We tested the AtAFB2-miniIAA7 system for rapidly depleting endogenous proteins and revealing acute phenotypes. Dynein heavy chain (DHC1) and epidermal growth factor receptor (EGFR) were chosen as the first targets. DHC1, a protein essential for cell division, suffered from severe basal degradation by using the OsTIR1miniAID system in a previous study ${ }^{8}$. EGFR is a transmembrane receptor with a canonical function in EGF uptake that can be acutely assessed after protein depletion. Endogenous target loci in human cells were tagged with miniIAA7-mEGFP (EGFP, enhanced green fluorescent protein) through Cas9-mediated homology-directed repair (HDR) (Fig. 1e). We tagged $\mathrm{DHC1}$ homozygously but could only tag EGFR heterozygously in A431 cells, likely due to its unusually high copy number in these cells (data not shown). However, we achieved homozygous tagging of EGFR in human A549 cells. AtAFB2 or OsTIR1 for comparison, was then introduced into the $A A V S 1$ locus of the homozygous knock-in clones. The parental cell lines not expressing an auxin receptor F-box protein were used as controls (Fig. 1e). We found that both DHC1-AtAFB2 cells (DHC1 homozygously tagged with miniIAA7-mEGFP and AtAFB2 expressing) and EGFR-AtAFB2 cells showed minimal basal degradation at $0 \mathrm{~h}$ IAA, and efficient auxin-inducible depletion at $1 \mathrm{~h}$ IAA and at longer times (Fig. If and Supplementary Fig. 4a). In comparison, DHC1-OsTIR1 cells died out during selection, in agreement with Natsume et al. ${ }^{8}$, and EGFR-OsTIR1 cells showed severe basal degradation at $0 \mathrm{~h}$ IAA (Fig. If and Supplementary Fig. 4a).

We next analyzed whether rapid auxin-inducible depletion revealed acute phenotypes. None of the mitotic rounding DHC1AtAFB2 cells managed to complete cell division after IAA addition for $30 \mathrm{~min}$, while all mitotic rounding cells completed cell division without IAA (Fig. $1 \mathrm{~g}$ and Supplementary Video 1). In EGFRAtAFB2 cells, EGF uptake was reduced by about $70 \%$ on $1 \mathrm{~h}$ IAA

'Department of Anatomy and Stem Cells and Metabolism Research Program, Faculty of Medicine, University of Helsinki, Helsinki, Finland. ${ }^{2}$ Minerva Foundation Institute for Medical Research, Helsinki, Finland. ${ }^{3}$ Department of Physics, University of Helsinki, Helsinki, Finland. ${ }^{4}$ Computational Physics Laboratory, Tampere University, Tampere, Finland. ${ }^{5}$ These authors contributed equally: Xavier Prasanna, Veijo T. Salo. ^e-mail: elina.ikonen@helsinki.fi 

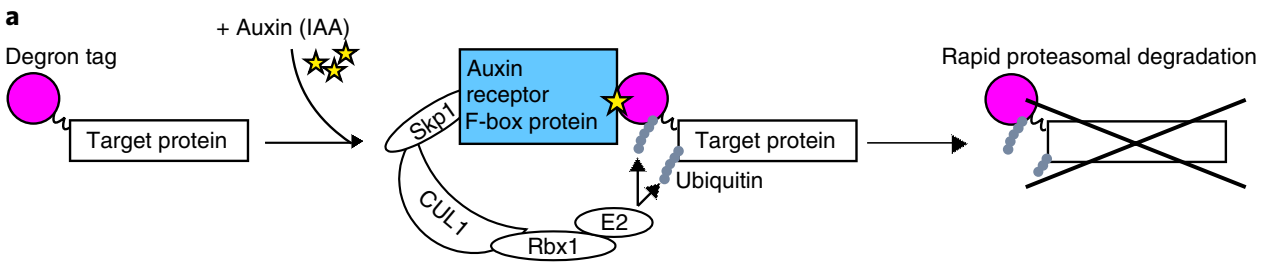

b
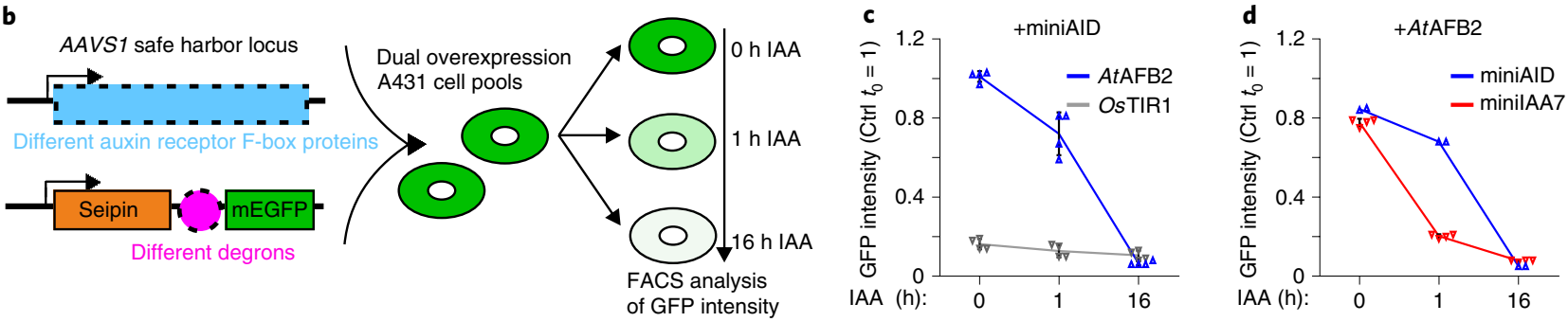

e
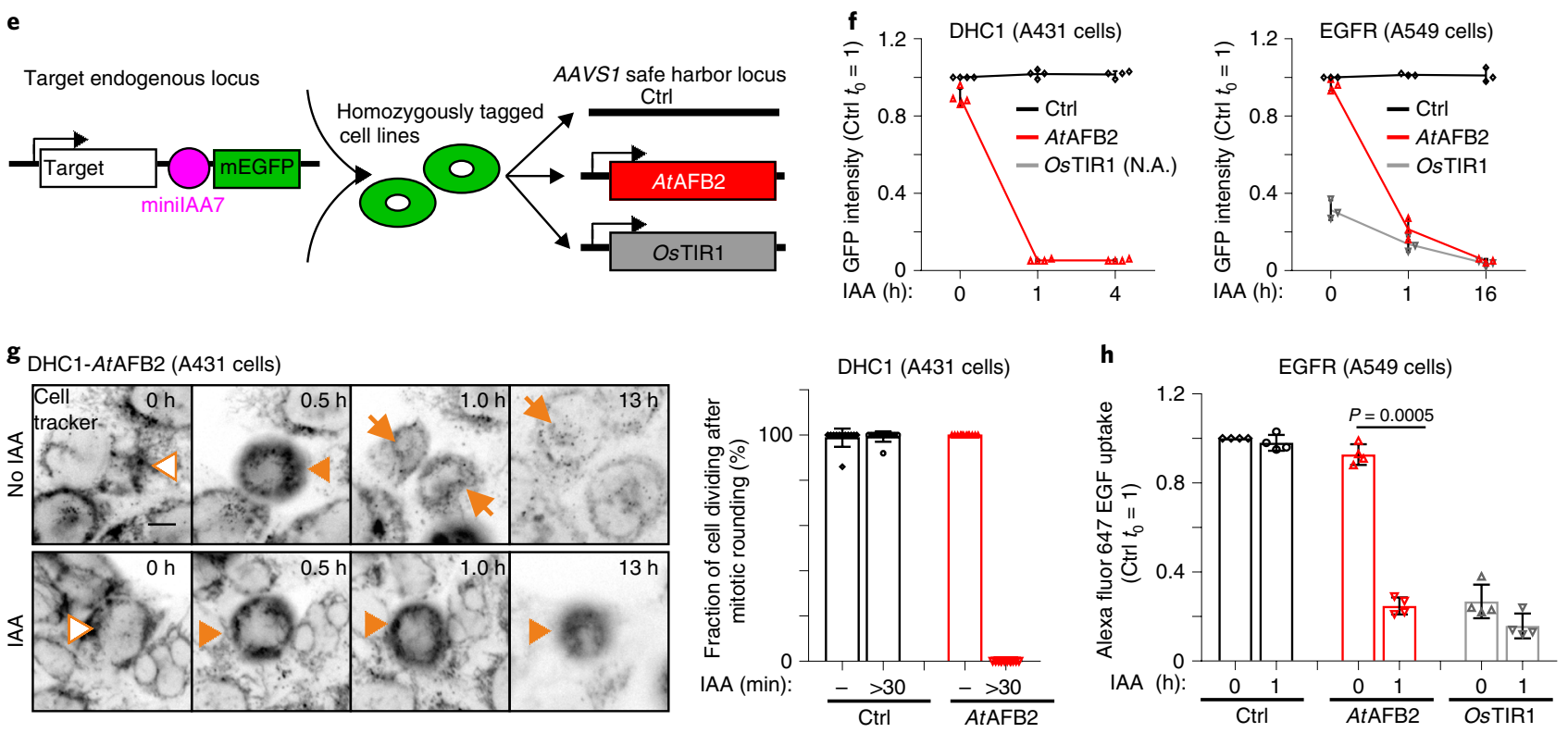

Fig. 1 Identification of AtAFB2-minilAA7 as an improved AID system. a, Principle of rapid protein depletion with AID. b, Scheme for screening auxin receptor F-box proteins and degrons. Target protein levels in cells are analyzed by FACS of GFP at $\mathrm{O}$ IAA for basal degradation, at $1 \mathrm{~h}$ IAA for early depletion efficiency and at $16 \mathrm{~h} \mathrm{IAA}$ for final depletion efficiency. c,d, Mean GFP intensity analyzed as shown in $\mathbf{b}$. c, miniAID as the degron. Bars represent mean \pm s.d.; $n=4$ biologically independent samples; ctrl, no auxin receptor F-box protein. d, AtAFB2 as the auxin receptor F-box protein. Bars represent mean \pm s.d.; $n=2$ biologically independent samples for miniAID, $n=4$ for minilAA7; ctrl, seipin-mEGFP without degron. e, Scheme for establishing cell lines with AtAFB2-minilAA7 system to deplete endogenous proteins. Control (ctrl) or OsTIR1 were used for comparison. f, Mean GFP intensity analyzed by FACS. Bars represent mean \pm s.d.; $n=4$ biologically independent samples for DHC1, $n=3$ for EGFR. N.A., not available due to inability to establish the cell line. $\mathbf{g}$, Time-lapse images of DHC1-AtAFB2 cells with or without cell dividing after mitotic cell rounding (left) and analysis of the fraction of cell dividing after mitotic rounding (right). Scale bar, $10 \mu \mathrm{m}$. On the left: open arrowhead, cells before mitosis; filled arrowhead, cells undergoing mitotic rounding; arrow, cells after cell division. On the right, bars represent mean \pm s.d.; $n=12$ fields. This experiment was repeated once independently with similar results. h, Alexa647 EGF uptake analyzed by FACS in EGFR cells. Bars represent mean \pm s.d.; paired two-tailed $t$-test; $n=4$ biologically independent samples.

treatment, while severe IAA-independent reduction of EGF uptake was observed in EGFR-OsTIR1 cells (Fig. 1h). The inducer IAA per se did not affect cell division or EGF uptake as shown in the controls (Fig. 1g,h and Supplementary Video 1). Overall, these results demonstrate the improved performance of the AtAFB2-miniIAA7 system in rapidly depleting endogenous proteins and revealing acute phenotypes in two human cell types.

In previous experiments, miniIAA7 was used as a $\mathrm{C}$ terminal tag (Fig. 1b,e). We next tagged miniIAA7 $\mathrm{N}$ terminally to an endogenous protein. We chose Sec61beta as it is a commonly used target for $\mathrm{N}$ terminal tagging through HDR. The $\mathrm{N}$ terminally tagged Sec61beta was efficiently depleted in $1 \mathrm{~h}$ with the AtAFB2-miniIAA7 system
(Supplementary Fig. 4b-e). The tag orientation miniIAA7-mEGFP improved auxin-inducible depletion over two-fold at $1 \mathrm{~h}$ IAA compared to mEGFP-miniIAA7 (Supplementary Fig. 4b-e). Thus, both $\mathrm{N}$ and $\mathrm{C}$ terminal tagging of miniIAA7 could rapidly deplete target proteins when using miniIAA7-mEGFP as a fixed unit.

We then evaluated the overall performance of the AtAFB2miniIAA7 system in depleting endogenous proteins. We tagged a diverse set of endogenous loci homozygously with miniIAA7mEGFP $\mathrm{N}$ or $\mathrm{C}$ terminally (Fig. 2a) and introduced AtAFB2 or OsTIR1 into the AAVS1 locus (as in Fig. 1e). The target proteins represented different subcellular localizations and a variable number of transmembrane segments, including our original target seipin 


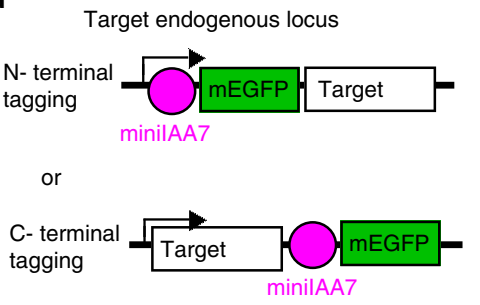

b

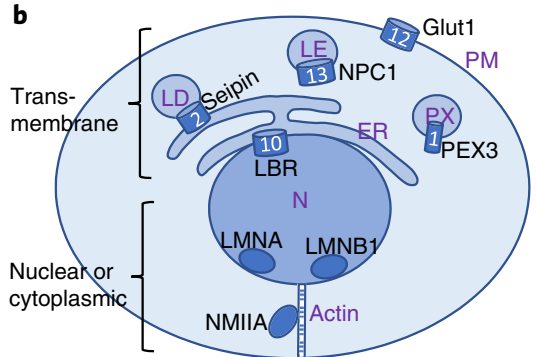

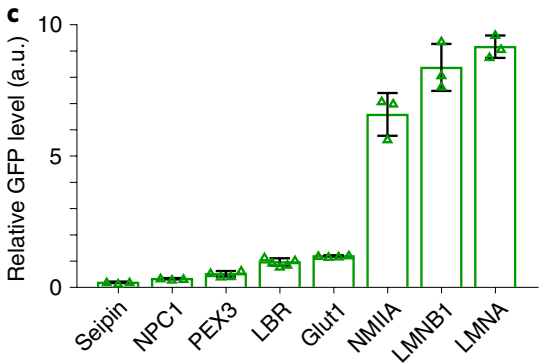
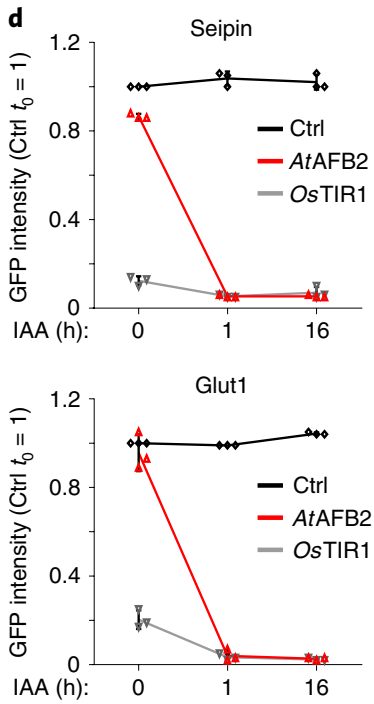
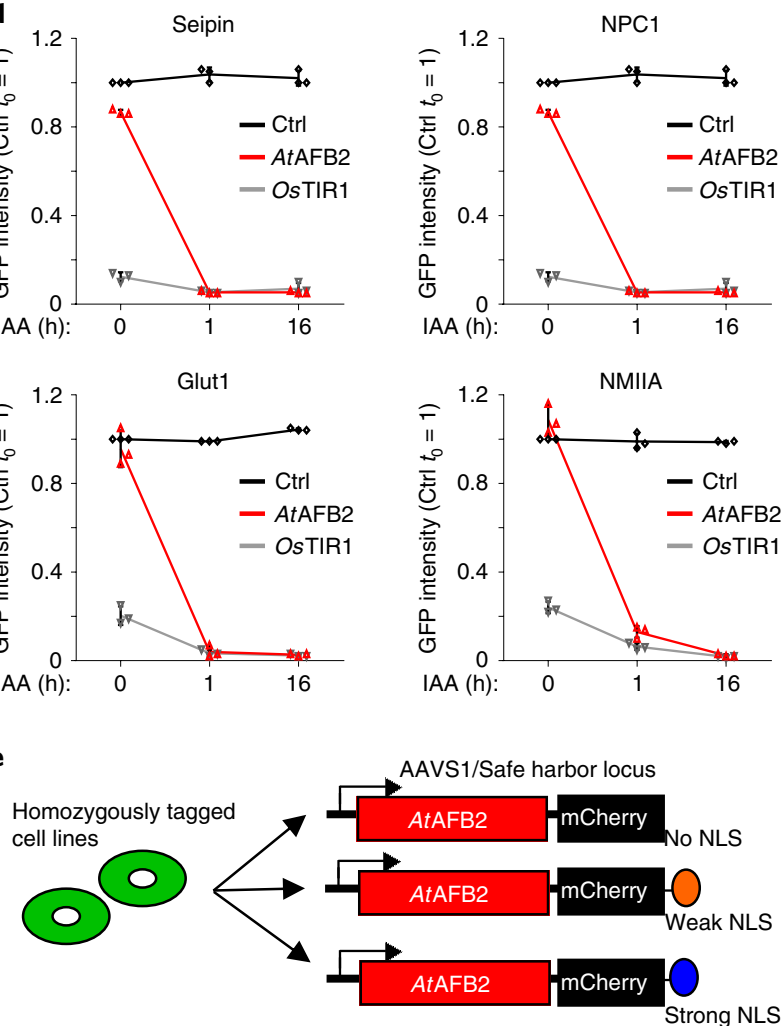
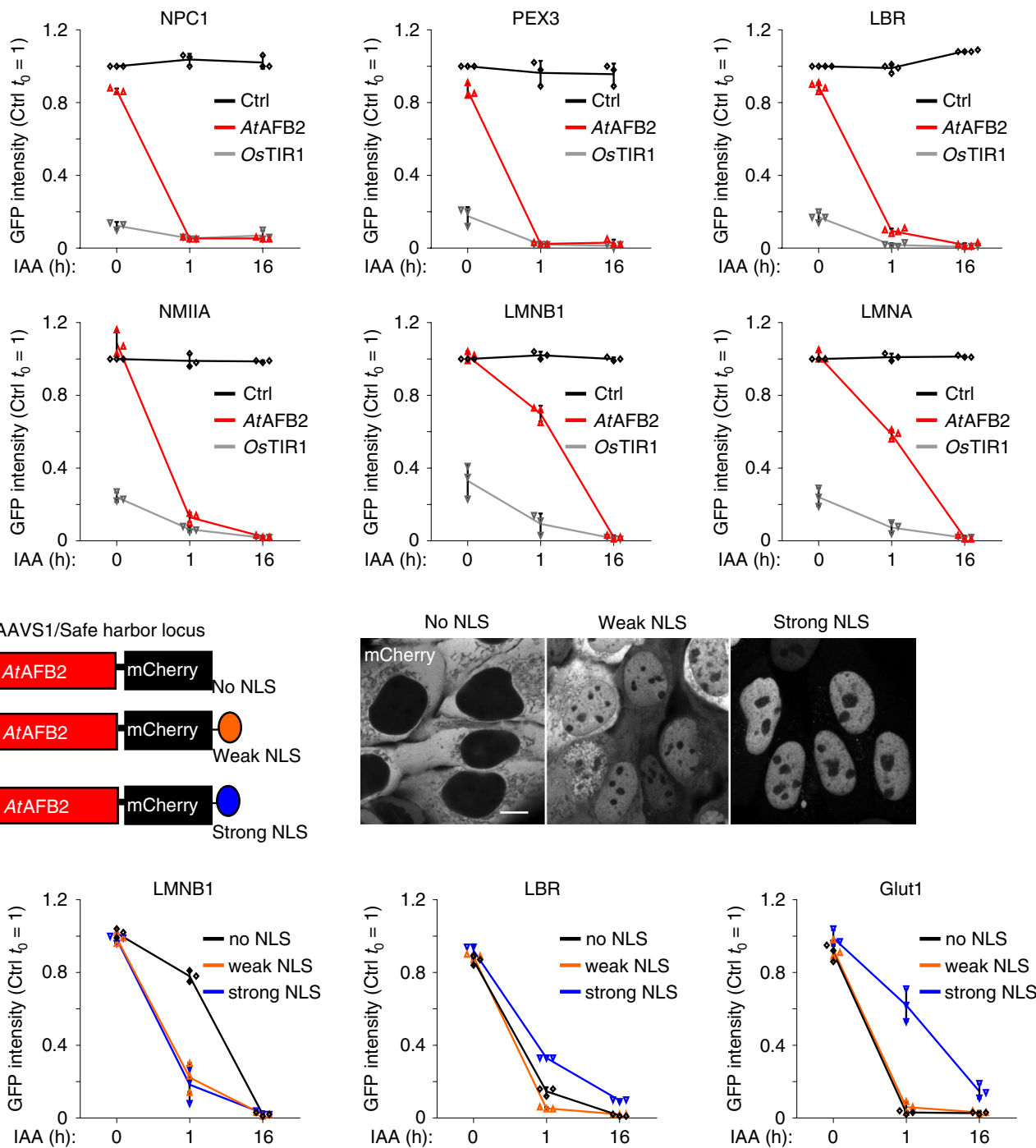

Fig. 2 | Depletion of endogenous transmembrane, cytoplasmic and nuclear proteins using the AtAFB2-minilAA7 system. a, Scheme for N or C terminal tagging of endogenous target locus with minilAA7-mEGFP. b, Illustration of subcellular localization of target proteins. Numbers represent transmembrane segments; ER, endoplasmic reticulum; LE, late endosome; N, nucleus; PM, plasma membrane; PX, peroxisome. c, Relative target protein levels analyzed by FACS of mean GFP intensity in ctrl cell lines at O h IAA. Bars represent mean \pm s.d.; $n=3$ biologically independent samples, except for Glut1 ( $n=4$ ), PEX3 $(n=4)$ and LBR $(n=5)$; a.u., arbitrary unit. d, Mean GFP intensity analyzed by FACS in cells with endogenously minilAA7-tagged targets and indicated auxin receptor F-box proteins or ctrl. Bars represent mean \pm s.d.; $n=3$ biologically independent samples, except for LBR ( $n=4$ ). e, Scheme for increasing AtAFB2 nuclear localization (left) and live cell Airyscan images of AtAFB2-mCherry without NLS, with weak or strong NLS in A431 cells (right). This experiment was repeated once independently with similar results. Scale bar, $10 \mu \mathrm{m}$. f, Mean GFP intensity analyzed by FACS in cells with endogenously minilAA7-tagged proteins and different AtAFB2-mCherry constructs. Bars represent mean \pm s.d.; $n=3$ biologically independent samples.

and a long-lived protein LMNB1 (ref. ${ }^{17}$ ) (Fig. 2b and Supplementary Fig. 5a). The expression levels of the target proteins in the established cell lines varied by $\sim 50$-fold (ranging from 0.19 for seipin to 9.21 for LMNA; Fig. 2c). When examining the performance of AtAFB2 with these targets in comparison to OsTIR1, we found that all targets had minimal basal degradation at $0 \mathrm{~h}$ IAA (86-109\% levels of controls) and were depleted to $2-5 \%$ levels at $16 \mathrm{~h}$ IAA (Fig. $2 \mathrm{~d}$ ). However, these targets showed variable depletion efficiency at $1 \mathrm{~h}$ IAA. 
Non-nuclear targets expressed at low levels (seipin, NPC1, PEX3 and Glut1) were depleted to $2-5 \%$. A non-nuclear target expressed at high level (NMIIA) and a target expressed at low level and localized mainly to the inner nuclear envelope (LBR) were also depleted to about $10 \%$. Instead, highly expressed nuclear proteins (LMNA and LMNB1) were not efficiently depleted (Fig. 2d). In comparison, the OsTIR1-miniIAA7 combination resulted in severe basal degradation with all targets at $0 \mathrm{~h}$ IAA (12-37\%) and unimproved depletion efficiency at $16 \mathrm{~h}$ IAA (2-7\%) (Fig. 2d).

We continued to improve the depletion of LMNA and LMNB1 using the AtAFB2-miniIAA7 system. Because AtAFB2 localized predominantly to the cytosol (Supplementary Fig. 2c), we increased the nuclear localization of AtAFB2-mCherry by fusing nuclear localization signals (NLSs) to it (Fig. 2e). Both weak and strong NLSs increased the nuclear localization of AtAFB2-mCherry and substantially improved auxin-inducible depletion of LMNA and LMNB1 at $1 \mathrm{~h}$ IAA (Fig. 2e,f). Of note, LBR not restricted to the nucleus, as well as Glut1 in the plasma membrane, showed efficient auxin-inducible depletion with the weak NLS but not with the strong NLS construct (Fig. 2f and Supplementary Fig. 4f). A single dose of IAA was sufficient for both non-nuclear and nuclear target depletion for $48 \mathrm{~h}$, and the expression of targets in the degron cells during normal culturing was stable (Supplementary Fig. 4g). In summary, our results demonstrate that the AtAFB2-miniIAA7 system rapidly depleted all selected endogenous transmembrane, cytoplasmic and nuclear proteins in $1 \mathrm{~h}$ with minimal basal degradation. For rapid protein depletion, AtAFB2 needs to be present at sufficient levels in the compartment where the target protein resides.

We found that depletion of the endogenous targets with the AtAFB2-miniIAA7 system revealed robust and expected phenotypes as early as $1 \mathrm{~h}$ after IAA addition, depending on the functional readouts. These included reduced glucose uptake in Glut1 degron cells (cells with AtAFB2-miniIAA7 system targeting Glut1), clear changes of F-actin structures in NMIIA degron cells, accumulation of cholesterol in late endosomal compartments in NPC1 degron cells, LD biogenesis defects in seipin degron cells, reduction of cellular cholesterol levels in LBR degron cells and extensive degradation of peroxisomal membrane proteins in PEX3 degron cells (Supplementary Fig. 5b-g).

MiniIAA7-mEGFP worked well for rapid inducible depletion in all the cases tested, but we also explored whether mEGFP can be omitted or substituted by other tags. We found that for seipin, $\mathrm{C}$ terminal miniIAA7 alone worked at a compromised efficiency and this could be improved by attaching mCherry, mScarlet-I (ref. ${ }^{18}$ ) or a small 3XFlag tag to its $C$ terminus (Supplementary Fig. 6a,b). We envision that mEGFP or other extensions to the $\mathrm{C}$ terminus of miniIAA7 may help to avoid steric hindrance during auxin-inducible binding and/or serve as an additional $\mathrm{SCF}^{\mathrm{AtAFB} 2} \mathrm{E} 3$ ligase substrate.

In comparison to the recently reported dTAG system ${ }^{19}$ (Supplementary Fig. 7a), the improved AID system may have some advantages. By employing AtAFB2 overexpression, the AID system is effective in different cell lines and subcellular compartments and for rapidly degrading proteins of high and low abundance. The dTAG system relies on the activity of endogenous cereblon (CRBN) and might be more host cell sensitive, as suggested by its low efficiency in A431 cells (Supplementary Figs. 7b,c and 8a,b). Moreover, rapid degradation of highly abundant proteins might be challenging with the dTAG system, as judged by the slower degradation of LMNA (Supplementary Fig. 8a,b).

Finally, protein degradation induced with the AtAFB2-miniIAA7 system was more readily reversible on IAA washout than with the OsTIR1-miniIAA7 system (Supplementary Fig. 9a,b). The improved AID system also appeared better than the dTAG system in washout experiments (Supplementary Figs. 7d and 9c), potentially because it employs a coreceptor strategy for IAA binding ${ }^{20}$ compared to the heterobifunctional binding of $\mathrm{dTAG}^{19}$ (Supplementary Fig. 9d).
Besides human cells, the AID technology has been applied in other organisms such as yeast ${ }^{2,4}$, Caenorhabditis elegans ${ }^{12}$, Drosophila ${ }^{13}$ and mouse embryos ${ }^{11}$. We believe that the AtAFB2-miniIAA7 system can also improve the performance of AID in these applications.

\section{Online content}

Any methods, additional references, Nature Research reporting summaries, source data, statements of code and data availability and associated accession codes are available at https://doi.org/10.1038/ s41592-019-0512-X.

Received: 7 March 2019; Accepted: 8 July 2019; Published online: 26 August 2019

\section{References}

1. Housden, B. E. et al. Nat. Rev. Genet. 18, 24-40 (2017).

2. Nishimura, K., Fukagawa, T., Takisawa, H., Kakimoto, T. \& Kanemaki, M. Nat. Methods 6, 917-922 (2009).

3. Tan, X. et al. Nature 446, 640-645 (2007).

4. Costa, E. A., Subramanian, K., Nunnari, J. \& Weissman, J. S. Science 359, 689-692 (2018)

5. Nora, E. P. et al. Cell 169, 930-944.e22 (2017).

6. Natsume, T. et al. Genes Dev. 31, 816-829 (2017)

7. Muhar, M. et al. Science 360, 800-805 (2018).

8. Natsume, T., Kiyomitsu, T., Saga, Y. \& Kanemaki, M. T. Cell Rep. 15, 210-218 (2016)

9. Daniel, K. et al. Nat. Commun. 9, 3297 (2018).

10. Yesbolatova, A., Natsume, T., Hayashi, K. \& Kanemaki, M. T. Methods 164-165, 73-80 (2019).

11. Gu, B., Posfai, E. \& Rossant, J. Nat. Biotechnol. 36, 632-637 (2018).

12. Zhang, L., Ward, J. D., Cheng, Z. \& Dernburg, A. F. Development 142, 4374-4384 (2015).

13. Bence, M., Jankovics, F., Lukácsovich, T. \& Erdélyi, M. FEBS J. 284, 1056-1069 (2017)

14. Kleinjan, D. A., Wardrope, C., Nga Sou, S. \& Rosser, S. J. Nat. Commun. 8, 1191 (2017).

15. Salo, V. T. et al. EMBO J. 35, 2699-2716 (2016).

16. Lombardo, A. et al. Nat. Methods 8, 861-869 (2011).

17. Toyama, B. H. et al. Cell 154, 971-982 (2013).

18. Bindels, D. S. et al. Nat. Methods 14, 53-56 (2017).

19. Nabet, B. et al. Nat. Chem. Biol. 14, 431-441 (2018).

20. Calderón Villalobos, L. I. A. et al. Nat. Chem. Biol. 8, 477-485 (2012).

\section{Acknowledgements}

We thank A. Uro for technical assistance and L. Kaipiainen for cholesterol measurements. HiLIFE Flow cytometry and Light microscopy platforms are acknowledged for access to research infrastructure. This study was supported by the Academy of Finland (Center of Excellence project grant no. 307415 to E.I. and I.V.; grant nos. 282192, 312491 to E.I.), the Sigrid Juselius Foundation (I.V. and E.I.), Finnish Medical Foundation (V.T.S.), Paulo Foundation (V.T.S.), Alfred Kordelin Foundation (V.T.S.), Maud Kuistila Foundation (V.T.S.), Biomedicum Helsinki Foundation (V.T.S.) and the Emil Aaltonen Foundation (V.T.S.)

\section{Author contributions}

E.I. and S.L. conceptualized the project. S.L. designed and performed AID screen and test experiments. S.L. and V.T.S. designed and performed functional studies. X.P. and I.V. provided theoretical and mechanistic insights. S.L. and E.I. wrote the manuscript with input from all authors. E.I. supervised the study.

\section{Competing interests}

A patent application covering the use of AtAFB2-miniIAA7 for rapid protein depletion (application no. FI 20195239) has been filed in which the University of Helsinki is the applicant, and E.I. and S.L. are the inventors.

\section{Additional information}

Supplementary information is available for this paper at https://doi.org/10.1038/ s41592-019-0512-x.

Reprints and permissions information is available at www.nature.com/reprints. Correspondence and requests for materials should be addressed to E.I.

Peer review information: Rita Strack was the primary editor on this article and managed its editorial process and peer review in collaboration with the rest of the editorial team.

Publisher's note: Springer Nature remains neutral with regard to jurisdictional claims in published maps and institutional affiliations.

(C) The Author(s), under exclusive licence to Springer Nature America, Inc. 2019 


\section{Methods}

Cell culture. A431 cells (ATCC, cat. no. CRL-1555) and HEK293A cells (Invitrogen, R70507) were cultured in DMEM (Lonza), and A549 cells (ATCC, cat. no. CCL-185) in F-12 Nutrient Mixture (Gibco), supplemented with $10 \%$ FBS, penicillin/streptomycin $\left(100 \mathrm{U} \mathrm{ml}^{-1}\right.$ each), $\mathrm{L}$-glutamine $(2 \mathrm{mM})$ at $37^{\circ} \mathrm{C}$ in $5 \% \mathrm{CO}_{2}$. Mycoplasma testing was performed regularly using PCR detection. Cells were transfected at $80-95 \%$ confluence using Lipofectamine LTX with PLUS Reagent (Life Technologies), typically with $1.0 \mu \mathrm{g}$ plasmid(s) per $1.0 \mu \mathrm{l}$ of PLUS reagent ( $1.5 \mu \mathrm{l}$ for HEK293A), $2.0 \mu$ l of Lipofectamine LTX (3.0 $\mu \mathrm{l}$ for HEK293A) and $4.0 \times 10^{5}$ (A431 and HEK293A) or $3.0 \times 10^{5}$ (A549) cells in a 12-well. Indole3 -acetic acid sodium (IAA, Santa Cruz, sc-215171) was prepared at $100 \times$ in $\mathrm{H}_{2} \mathrm{O}$ $\left(10 \mathrm{mg} \mathrm{ml}^{-1}\right)$, aliquoted, stored at $-20^{\circ} \mathrm{C}$ and used within $2 \mathrm{~d}$ after thawing.

\section{Construction of vectors for AAVS1 site-specific integration. AAVS1 safe} harbor locus site-specific integration was conducted with CRISPR/Cas9mediated HDR. A donor vector was generated by assembling PCR amplified fragments by restriction digestion and ligation. The resulting vector contained two homology arms (from A431 genomic DNA) flanking an overexpression cassette with puromycin selection marker (from pEFIRES- ${ }^{21}$ ) on a plasmid backbone (from pGL3-basic). This donor vector was designated as $\mathrm{pSH}$ EFIRES-P and used to express different auxin receptor F-box proteins. A second donor vector was generated by changing the puromycin selection marker on the first vector with blasticidin selection marker. This donor vector was designated as pSH-EFIRES-B and used to express seipin-mEGFP with different degrons. A third vector co-expressing Cas9 and a single guide RNA (sgRNA) (both derived from PX458, Addgene no. 48138, ref. ${ }^{22}$ ) was designated as pCas9-sgRNA. The vector was inserted with two sgRNAs targeting AAVS1 safe harbor locus (sgAAVS1-1 target sequence: ACCCCACAGTGGGGCCACTA GGG; sgAAVS1-2 target sequence: GTCACCAATCCTGTCCCTAG TGG). Auxin receptor F-box proteins, except OsTIR1 (Addgene no. 72835), and degron tags were codon-optimized and synthesized by Genscript (sequences in Supplementary Table 1). Auxin receptor F-box proteins were tagged with mCherry through overlap PCR using a 5 aa linker GGSGG. The two NLSs for AtAFB2-mCherry were weak NLS (MycA1, AAAKRVKLD) and strong NLS (Myc, PAAKRVKLD). The three vectors with insertions and their full sequences will be deposited in Addgene.

Generation of A431 cell pools for screening. A431 cell pools were generated to stably express different combinations of auxin receptor F-box protein and degron-fused seipin through co-integration into the AAVS1 safe harbor locus. Cells were cotransfected with a mixture of three vectors composed of pSH-EFIRES-P expressing an auxin receptor F-box protein, pSH-EFIRES-B expressing a degronfused seipin and pCas9-sgAAVS1 at ratio of 3:3:4. Transfected cells were passaged 4-6h after transfection at 1:5 into six-well plates. The next day, cells were selected with $1 \mu \mathrm{g} \mathrm{ml}^{-1}$ puromycin (Sigma, P8833) for $2 \mathrm{~d}$, and with $5 \mu \mathrm{g} \mathrm{ml}^{-1}$ blasticidin (Gibco, A1113904) for $2 \mathrm{~d}$, then with both antibiotics for at least $6 \mathrm{~d}$ before using for fluorescence-activated cell sorting (FACS) analysis.

FACS analysis. Cells were seeded at 1:5 (for A431) or 1:3 (for A549) into a six-well plate in medium without selection on day 0 . On day 1 , the medium was changed to $2 \mathrm{ml}$ fresh medium without (for $0 \mathrm{~h}$ and $1 \mathrm{~h}$ IAA samples) or with (for $16 \mathrm{~h}$ IAA samples) $0.5 \mathrm{mM}$ IAA. On day 2 , the $1 \mathrm{~h}$ samples were supplemented with $0.5 \mathrm{ml}$ medium containing $2.5 \mathrm{mM}$ IAA (final $0.5 \mathrm{mM}$ ) and incubated for $1 \mathrm{~h}$ at $37^{\circ} \mathrm{C}$. After treatment, cells were detached with $0.5 \mathrm{ml}$ trypsin at $37^{\circ} \mathrm{C}$ for $5-8 \mathrm{~min}$ (A549) or 8-12 $\mathrm{min}$ (A431), put on ice and transferred to $1.5 \mathrm{ml}$ Eppendorf tubes containing $0.5 \mathrm{ml}$ serum-free $\mathrm{CO}_{2}$ independent medium (Gibco). The cell suspensions were centrifuged at $4{ }^{\circ} \mathrm{C}$, resuspended in $0.3 \mathrm{ml}$ ice-cold serum-free FluoroBrite DMEM (Gibco) and stored on ice before FACS analysis. FACS analysis was performed on a BD Influx cell sorter (BD Biosciences) with a $100 \mu \mathrm{m}$ nozzle at $4-8^{\circ} \mathrm{C}$ using BD FACS Sortware. Cells were gated with SSC, FSC and trigger pulse width for singlets, and 100,000 cells were analyzed from each sample. GFP was excited with a $488 \mathrm{~nm}$ laser and detected with a 530/40 detector; mCherry was excited with a $561 \mathrm{~nm}$ laser and detected with a 615/20 detector. Data were analyzed with BD FACS Sortware. Background subtracted mean fluorescence intensity was used for analysis.

Construction of vectors for endogenous tagging. Degron tagging of endogenous loci was conducted with CRISPR-Cas9-mediated HDR. Donor vectors with two homology arms flanking the degron tag, and Cas9 vectors with specific sgRNAs were constructed for each target. For constructing donor vectors, homology arms were amplified from A431 genomic DNA. MiniIAA7-mEGFP tags were amplified from established templates in the screens above and miniAID tag (used in Supplementary Fig. 1) was amplified from Addgene no. 72825 (ref. ${ }^{8}$ ). Overlap PCR was then performed to assemble PCR fragments. Nested PCR primers were used to improve the PCR efficiency and specificity. All PCR amplification steps were performed with Q5 Hot Start High-Fidelity DNA Polymerase (NEB). The PCR fragments were cloned into plasmid backbones using HiFi DNA assembly kit (NEB) or through restriction ligation. Some of the donor vectors were generated by changing the inserts on the established donor vectors through restriction ligation. For constructing Cas9 vectors, target sites were searched manually for -NGG PAM sequence within 18 base pairs after insertion sites or CCN- PAM within $18 \mathrm{bp}$ before insertion sites. These position restraints were set for both high-efficiency integration and for avoiding further mutations after successful HDR. The DHC1 target sites were selected at the $3^{\prime}$ untranslated region, as no target site was available in the searching range. A pCas9/QRVR-sgRNA ${ }^{23}$ vector was later constructed through PCR mutagenesis of pCas9-sgRNA to enable use of target sites with -NGA (or TCN-) PAM in the searching range. SgRNAs were synthesized as two unphosphorylated primers, annealed and inserted into BbsI-cut pCas9-sgRNA or pCas9/VRQR-sgRNA vector. Information about endogenous targets, HDR templates, primers for HDR templates and sgRNAs is provided in Supplementary Table 1.

Generation of homozygously tagged cell lines. HDR pools were first generated, followed by FACS enrichment of high GFP expressing cells and limiting dilution in 96-well plates to obtain single clones. Single clones were screened first by fluorescence microscopy for proper GFP expression and subcellular localization, then by genomic PCR to check for homozygous tagging. A detailed protocol is described below. A431 or A549 cells in 12-well plates were first transfected with a donor vector $(0.6 \mu \mathrm{g})$ plus a Cas $9 / \mathrm{sgRNA}$ vector encoding puromycin resistance gene $(0.4 \mu \mathrm{g})$. After $4-6 \mathrm{~h}$, cells were passaged into $10 \mathrm{~cm}$ dishes. The next day, medium was changed to $1 \mu \mathrm{g} \mathrm{ml}^{-1}$ puromycin for $2 \mathrm{~d}$, then to normal medium without puromycin. This procedure eliminated efficiently untransfected cells without selecting for stable puromycin resistant cells. After culturing in normal medium for $4 \mathrm{~d}$, the cells were passaged to fresh medium for $2 \mathrm{~d}$ and the resulting cells were considered the HDR pools. For each target, we typically tried 2-3 sgRNAs in duplicate, and the relative HDR efficiencies in the pools were assessed by fluorescence microscopy. HDR pools with the highest efficiency were used for FACS analysis as above, and cells with the highest $1-5 \%$ GFP intensity were gated for sorting. The sorted cells were used for single clone isolation with limiting dilution in 96-well plates. For each pool, 10-20 clones were isolated, from which 2-3 clones were picked with fluorescent microscopy for high GFP signal and proper subcellular localization. These clones were further tested for homozygous tagging using genomic PCR, with typically two single clones finally selected for each target. The best sgRNAs, their efficiency in HDR pools analyzed by FACS and primers for genomic PCR are listed in Supplementary Table 1.

Generation of degron cell lines. Homozygously tagged single clones were used to generate degron cells overexpressing an auxin receptor F-box protein. Auxin receptor F-box proteins were introduced into the AAVS1 safe harbor loci of single clones through Cas9-mediated HDR. Cells were transfected with $0.4 \mu \mathrm{g}$ pCas9sgAAVS1 and $0.6 \mu \mathrm{g}$ pSH-EFIRES-P plasmid encoding an auxin receptor F-box protein. Transfected cells were passaged at 1:5 after $4-6 \mathrm{~h}$. The next day, cells were selected with $1 \mu \mathrm{g} \mathrm{ml}^{-1}$ puromycin for $6 \mathrm{~d}$ before passaging for experiments or further culturing. The resulting cell pools were used for FACS analysis and lossof-function studies without single cloning. Then, $5 \mu \mathrm{g} \mathrm{ml}^{-1}$ of puromycin was used occasionally to improve the expression level of the auxin receptor F-box proteins in the A431 pools. FACS sorting was performed to enrich A549-EGFR pools responsive to IAA treatment. For this, A549 pools were treated with $1 \mathrm{~h}$ IAA and sorted for cells with low GFP.

Live cell Airyscan imaging. Cells cultured in FluoroBrite DMEM with 10\% FBS in eight-well Lab-Tek II no. 1.5 coverglass slides (Thermofisher) were imaged with a Zeiss LSM 880 equipped with an Airyscan detector using a $\times 63$ Plan-apochromat oil objective with a numerical aperture of 1.4. Live cell imaging was performed at $37^{\circ} \mathrm{C}, 5 \% \mathrm{CO}_{2}$ with incubator insert PM S1. Images were Airyscan processed automatically using the Zeiss ZEN2 software package.

\section{Analysis of cell division in A431 cells with tagged DHC1. Cells were plated} on $\mu$-slide eight-well ibiTreat dishes at $0.1 \times 10^{5}$ cells per well $2 \mathrm{~d}$ before the experiment. On the experiment day, cells were loaded with $2 \mu \mathrm{M}$ CellTracker Red CMTPX (Thermo, cat. no. C34552) in complete medium for 15-30 min at $37^{\circ} \mathrm{C}$. Medium was then changed to FluroBrite DMEM containing $10 \%$ FBS and incubated at $37^{\circ} \mathrm{C}$ for $1-2 \mathrm{~h}$ before imaging to equilibrate the label. Cells were imaged with Nikon Eclipse Ti-E microscope equipped with $\times 20$ air objective, numerical aperture of 0.8, Nikon Perfect Focus System 3, Hamamatsu Flash 4.0 v.2 scientific complementary metal-oxide-semiconductor and Okolab stage top incubator system. Before recording, six fields for each of the eight wells were selected with CellTracker Red fluorescence. IAA was then added at a final $0.5 \mathrm{mM}$ concentration to IAA-treated samples, mixed well with pipetting and time-lapse imaging started immediately recording every $30 \mathrm{~min}$ for $16 \mathrm{~h}$. Mitotic rounding cells were counted manually in the videos from $0.5 \mathrm{~h}$ to $6 \mathrm{~h}$. Mitotic rounding cells without cell division were followed until $13 \mathrm{~h}$ had elapsed.

EGF uptake in A549 cells with tagged EGFR. A549 cells were seeded at $0.4 \times 10^{5}$ cells per four wells for $3 \mathrm{~d}$. Medium was changed to fresh medium with or without $0.5 \mathrm{mM}$ IAA for $1 \mathrm{~h}$. Cells were washed twice with ice-cold serum-free medium with $1 \% \mathrm{BSA}$, and $0.2 \mathrm{ml}$ of $2 \mu \mathrm{g} \mathrm{ml}^{-1}$ Alexa Fluor 647 EGF complex (ThermoFisher, 
E35351) in serum-free medium with $1 \%$ BSA was added. Cells were further incubated at $37^{\circ} \mathrm{C}$ for $20 \mathrm{~min}$ before collecting with trypsin. Samples were kept on ice before FACS analysis. Alexa Fluor 647 was excited with $640 \mathrm{~nm}$ laser and detected with 720/40 detector, analyzing 20,000 cells per sample. Negative control samples were cells incubated in medium without EGF complex. Background subtracted mean fluorescence intensity was used for analysis.

Glucose uptake in A431 cells with tagged Glut1. Cells were plated at $0.6 \times 10^{5}$ cells on four-well plates $2 \mathrm{~d}$ before the experiment. On the experiment day, cells were treated with or without $0.5 \mathrm{mM}$ IAA for $1 \mathrm{~h}$ at $37^{\circ} \mathrm{C}$, then washed with DPBS (Gibco, 14040117 with calcium and magnesium). Glucose uptake was measured by incubating cells with $1 \mathrm{mM} 2$-deoxyglucose in DPBS for $10 \mathrm{~min}$ at room temperature and subsequent steps were performed according to the manufacturer's protocol (Promega, cat. no. J1341). Luminescent signal was measured in a 96 black well microplate (SCREENSTAR, cat. no. 655866) with VICTOR X3 multimode plate reader (PerkinElmer). Cells incubated with DPBS only were used as background. Protein concentrations were measured with Bio-Rad DC assay. Glucose uptake after background subtraction was normalized to protein concentration.

F-actin staining in A431 cells with tagged NMIIa. Cells were plated at $0.3 \times 10^{5}$ on $\mu$-slide eight-well ibiTreat chambers $1 \mathrm{~d}$ before the experiment. On the experiment day, cells were treated with or without $0.5 \mathrm{mM}$ IAA for $2 \mathrm{~h}$ at $37^{\circ} \mathrm{C}$, then washed with PBS, fixed with $4 \%$ PFA in $250 \mathrm{mM}$ HEPES, pH 7.4, $100 \mu \mathrm{M} \mathrm{CaCl}_{2}$ and $100 \mu \mathrm{M}$ $\mathrm{MgCl}_{2}$ for $20 \mathrm{~min}$, followed by quenching in $50 \mathrm{mM} \mathrm{NH}_{4} \mathrm{Cl}$ for $10 \mathrm{~min}$ and three washes with PBS. Cells were then stained with $0.132 \mu \mathrm{M}$ Alexa Fluor 568 Phalloidin (Molecular probes A-12380) in PBS for $30 \mathrm{~min}$ at room temperature. Z-stacks spanning the whole cell (step size $0.3 \mu \mathrm{m}$ ) were acquired with Nikon Eclipse Ti-E microscope, 60X PlanApo VC oil objective with a numerical aperture of 1.4 and a $\times 1.5$ zoom. Image stacks were automatically deconvolved using the Huygens batch processing application (Scientific Volume Imaging), and deconvolved image stacks were maximum intensity projected in ImageJ FIJI.

Filipin staining in A431 cells with tagged NPC1. Cells on coverslips in complete medium were treated with or without $0.5 \mathrm{mM}$ IAA for $16 \mathrm{~h}$, fixed and quenched as above. Fixed cells were then stained with $50 \mu \mathrm{g} \mathrm{ml}^{-1}$ of filipin in PBS for $30 \mathrm{~min}$ at $37^{\circ} \mathrm{C}$. Cells were washed twice with PBS and mounted with mowiol-DABCO. Imaging was performed on a Nikon Eclipse Ti-E microscope equipped with $\times 100$ oil objective with a numerical aperture of 1.4.

LD biogenesis in A431 cells with tagged seipin. Cells were delipidated by culturing in serum-free medium supplemented with $5 \%$ lipoprotein-deficient serum for $3 \mathrm{~d}$ and treated with or without $0.5 \mathrm{mM}$ IAA for the final $16 \mathrm{~h}$ on $\mu$-slide eight-well ibiTreat slides. For LD biogenesis, cells were loaded with $0.2 \mathrm{mM}$ oleic acid (oleic acid prepared as a $1 \mathrm{mM}$ OA-BSA complex at a 10:1 molar ratio to BSA in serum-free DMEM) for the final $2 \mathrm{~h}$, fixed and quenched as above. LDs were stained with LD540 (synthesized by Princeton BioMolecular Research, $0.1 \mu \mathrm{g} \mathrm{ml}^{-1}$ ) and nuclei with DAPI (Sigma, D9542, $10 \mu \mathrm{g} \mathrm{ml}^{-1}$ ). Z-stacks spanning the whole cell (step size $0.3 \mu \mathrm{m}$ ) were acquired with Nikon Eclipse Ti-E microscope, $\times 60$ PlanApo VC oil objective with a numerical aperture of 1.4 and a $\times 1.5$ zoom lens, and image stacks were automatically deconvolved using the Huygens batch processing application (Scientific Volume Imaging), then deconvolved image stacks maximum intensity projected by custom MATLAB scripts. Cell segmentation, LD detection and LD size distribution analysis was performed with CellProfiler ${ }^{24}$ and custom MATLAB software was generated for post-processing.

Cholesterol measurement in A431 cells with tagged LBR. Cells were delipidated by culturing in serum-free medium supplemented with $5 \%$ lipoprotein-deficient serum for $3 \mathrm{~d}$ and treated with or without $0.5 \mathrm{mM}$ IAA for the final $48 \mathrm{~h}$. Cells were washed, gathered with ice-cold PBS and cell pellets were used for analysis. Cholesterol was measured by gas-liquid chromatography analysis. The chloroformmethanol extracts of cellular lipids were saponified with potassium hydroxide in ethanol, extracted with hexane and silylated with trichloromethylsilane.

Cholesterol was separated from noncholesterol sterols and squalene and quantified by capillary gas-liquid chromatography with flame ionization detection and using a 50 -m capillary column (Ultra 2 , Agilent Technologies) with $5 \alpha$-cholestane as the internal standard as described ${ }^{25}$. Protein concentration was measured from aliquots of the same samples with a Bio-Rad DC Protein assay.

Western blotting. Cells were lyzed in buffer containing 1.0\% Igepal CA-630, 0.5\% sodium deoxycholate, $0.1 \%$ sodium dodecyl sulfate, $250 \mathrm{mM}$ Tris- $\mathrm{HCl}$, pH 7.5 and $150 \mathrm{mM} \mathrm{NaCl}$ with protease inhibitors. Samples in $1 \times$ Laemmli buffer were incubated at $37^{\circ} \mathrm{C}$ for $0.5-2 \mathrm{~h}$ before loading. Equal amounts of protein (measured using DC Protein assay) were loaded onto 7.5-12\% TGX Stain-Free gels, activated after running and transferred onto LF PVDF membrane (Bio-Rad). Images of Stain-Free total protein staining were acquired after transferring to PVDF membrane. Membranes were blotted with Odyssey blocking buffer (LI-COR) at room temperature for $0.5-1.0 \mathrm{~h}$, incubated with first antibody (rabbit anti-GFP, Abcam ab290; mouse anti-alpha Tubulin, Sigma B-5-1-2; mouse anti-EGFR, DSHB
CPTC-EGFR-1; mouse anti-PMP70, Sigma SAB4200181; rabbit anti-seipin, inhouse-generated antibody against $C$ terminal region of human seipin; mouse anti$\mathrm{HA}$, Sigma H9658) at $4^{\circ} \mathrm{C}$ overnight or at room temperature for $2 \mathrm{~h}$. Detection was performed with IRDye 800CW goat anti-mouse (Li-cor 926-32210) and Alexa 680 goat anti-rabbit antibodies (Invitrogen A21109) and all images were acquired with a ChemiDoc MP Imaging System (Bio-Rad).

Analysis of PMP22-mCardinal fluorescence in A431 cells with tagged PEX3. Cells were transfected with mCardinal-PMP-N-10 (Addgene no. 56173, a gift from M. Davidson $)^{26}$. Single clones with low mCardinal-PMP-N-10 expression and proper subcellular localization were isolated after FACS sorting of low mCardinal fluorescent cells. Cells were treated with or without $0.5 \mathrm{mM}$ IAA for $14 \mathrm{~d}$ and seeded on $\mu$-slide eight-well ibiTreat chambers for the final $2 \mathrm{~d}$. Cells were fixed and quenched as above. Nuclei were stained with DAPI (Sigma, D9542, $10 \mu \mathrm{g} \mathrm{ml}^{-1}$ ). Z-stacks spanning the whole cell (step size $0.3 \mu \mathrm{m}$ ) were acquired with Nikon Eclipse Ti-E microscope, $\times 60$ PlanApo VC oil objective with a numerical aperture of 1.4 and $\times 1.5$ zoom lens. Maximum intensity projections were generated in FIJI and cells segmented in CellProfiler. Background subtracted PMP22-mCardinal fluorescence intensity was analyzed from the segmented images using a custom MATLAB software generated for post-processing.

Testing different miniIAA7 tags. For testing of different miniIAA7 tags, a single A431 cell clone stably overexpressing AtAFB2-mCherry was first isolated. Constructs overexpressing seipin with different miniIAA7 fusion tags were generated through PCR and restriction ligation (mScarlet-I from pLifeAct mScarlet-i_N1, Addgene no. 85056, ref. ${ }^{18}$ ). These constructs were introduced into the AAVS1 safe harbor locus of AtAFB2-mCherry overexpressing cell clone through CRISPR-Cas9-mediated HDR as described above. After selection, stable cell pools were used for experiments. The full sequences and the plasmids encoding miniIAA7-mCherry, miniIAA7-mScarlet-I and miniIAA7-3XFlag will be deposited in Addgene.

Testing the dTAG system. Endogenous BRD4 in HEK293A and A431 cells was tagged $\mathrm{N}$ terminally with FKBP (F36V) through CRISPR-Cas9-mediated Precise Integration into Target Chromosome (PITCh) as reported ${ }^{19}$. Briefly, cells in 12-wells were transfected with $0.6 \mu \mathrm{g}$ of template pCRIS-PITChv2BSD-dTAG (BRD4) (Addgene no. 91792) plus $0.4 \mu \mathrm{g}$ of sgRNAs/Cas9 plasmid pX330A-nBRD4/PITCh (Addgene no. 91794) with Lipofectamine LTX. At $4-6 \mathrm{~h}$ post-transfection, cells were passaged to $25 \mathrm{~cm}^{2}$ dishes for $2 \mathrm{~d}$. Cells were further passaged to $60 \mathrm{~cm}^{2}$ dishes and selected with $10 \mu \mathrm{g} \mathrm{ml}^{-1}$ blasticidin (Gibco, A1113904) for $6 \mathrm{~d}$ before passaging for experiments or further culturing. Endogenous LMNA in cells was tagged N terminally with FKBP (F36V) through CRISPR-Cas9-mediated HDR. MiniIAA7-mEGFP tag in LMNA HDR template was substituted through restriction ligation with Puro-P2A-2XHA-FKBP (F36V) tag (from pCRIS-PITChv2-Puro-dTAG (BRD4), Addgene no. 91793). Cell pools were generated by transfection with $0.6 \mu \mathrm{g}$ of template plus $0.4 \mu \mathrm{g}$ of pCas9sgLMNA. Transfected cells were passaged and selected as above, except using $2 \mu \mathrm{g} \mathrm{ml}^{-1}$ puromycin for selection. The bifunctional degrader dTAG-13 (TOCRIS, $6605)$ was prepared as $20 \mathrm{mM}$ stock in DMSO $\left(21 \mathrm{mg} \mathrm{ml}^{-1}\right)$, aliquoted and stored at $-20^{\circ} \mathrm{C}$. The stock was further diluted with DMSO so that for all treatments, DMSO was used at $0.02 \%$ final concentration.

HDR coselection of LMNA degron cell pools. Cells with endogenously tagged LMNA were coselected by selecting cells with AtAFB2-mCherryweak NLS integration into AAVS1 locus, as both happen through $\mathrm{HDR}^{27}$ Cells in a 12-well were transfected with three plasmids, pCas9/sgLMNA/ sgAAVS1, LMNA HDR template plasmid and AtAFB2-mCherry-weak NLS in pSH-EFIRES-P at a ratio of 4:4.5:1.5. Cells were passaged to a $60 \mathrm{~cm}^{2}$ dish at $4-5 \mathrm{~h}$ post-transfection and selected with $2 \mu \mathrm{g} \mathrm{ml}^{-1}$ puromycin starting on the next day for $2 \mathrm{~d}$, followed by $5 \mu \mathrm{g} \mathrm{ml}^{-1}$ puromycin for $4 \mathrm{~d}$. The resulting cell pools were used for experiments.

Reversibility assays. For washout experiments, cells were seeded at day 1. Cells were treated with $0.5 \mathrm{mM}$ IAA or $0.1 \mu \mathrm{M}$ dTAG-13 (Supplementary Fig. 9a-c) or $0.5 \mu \mathrm{M}$ dTAG-13 (Supplementary Fig. $7 \mathrm{~d}$ ) for $16 \mathrm{~h}$ at different time points, washed with complete medium twice and further cultured for indicated periods before collection at day 4 for FACS or western blot analysis.

Statistics and reproducibility. Graphpad Prism 7.04 (Graphpad Software, Inc.) was used to generate graphs, perform $t$-tests and calculate $P$ values. Quantitative data are presented as mean \pm s.d., if not otherwise stated. The raw data underlying the graphical representations are provided in the Source data. Typically, two cell lines were used for endogenous targets. All immunoblots are representatives of two independent experiments. The uncropped scans of all immunoblots shown are provided in Supplementary Fig. 10.

Reporting Summary. Further information on research design is available in the Nature Research Reporting Summary linked to this article. 


\section{Data availability}

The data that support the findings of this study are available from the corresponding author upon reasonable request.

\section{Code availability}

Custom software scripts (to be used with CellProfiler and MATLAB in Supplementary Fig. 5e,g) can be found at https://bitbucket.org/szkabel/ lipidanalyser/get/master.zip.

\section{References}

21. Hobbs, S., Jitrapakdee, S. \& Wallace, J. C. Biochem. Biophys. Res. Commun. 252, 368-372 (1998).

22. Ran, F. A. et al. Nat. Protoc. 8, 2281-2308 (2013).

23. Kleinstiver, B. P. et al. Nature 529, 490-495 (2016).

24. Carpenter, A. E. et al. Genome Biol. 7, R100 (2006)

25. Miettinen, T. A. J. Lipid Res. 29, 43-51 (1988).

26. Chu, J. et al. Nat. Methods 11, 572-578 (2014).

27. Agudelo, D. et al. Nat. Methods 14, 615-620 (2017). 


\section{natureresearch}

Corresponding author(s): Elina Ikonen

Last updated by author(s): Jun 24, 2019

\section{Reporting Summary}

Nature Research wishes to improve the reproducibility of the work that we publish. This form provides structure for consistency and transparency in reporting. For further information on Nature Research policies, see Authors \& Referees and the Editorial Policy Checklist.

\section{Statistics}

For all statistical analyses, confirm that the following items are present in the figure legend, table legend, main text, or Methods section.

n/a Confirmed

\The exact sample size $(n)$ for each experimental group/condition, given as a discrete number and unit of measurement

$\bigotimes$ A statement on whether measurements were taken from distinct samples or whether the same sample was measured repeatedly

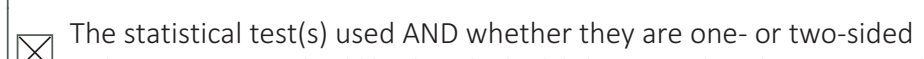

Only common tests should be described solely by name; describe more complex techniques in the Methods section.

$\triangle$ A description of all covariates tested

$\square$ A description of any assumptions or corrections, such as tests of normality and adjustment for multiple comparisons

A full description of the statistical parameters including central tendency (e.g. means) or other basic estimates (e.g. regression coefficient)

$\triangle$ AND variation (e.g. standard deviation) or associated estimates of uncertainty (e.g. confidence intervals)

For null hypothesis testing, the test statistic (e.g. $F, t, r$ ) with confidence intervals, effect sizes, degrees of freedom and $P$ value noted

$\triangle$ Give $P$ values as exact values whenever suitable.

Х $\square$ For Bayesian analysis, information on the choice of priors and Markov chain Monte Carlo settings

Х $\square$ For hierarchical and complex designs, identification of the appropriate level for tests and full reporting of outcomes

Х $\square$ Estimates of effect sizes (e.g. Cohen's $d$, Pearson's $r$ ), indicating how they were calculated

Our web collection on statistics for biologists contains articles on many of the points above.

\section{Software and code}

\section{Policy information about availability of computer code}

Data collection BD FACS Sortware for FACS data.

Zeiss ZEN2 software package for Airyscan images.

NIS-Elements Advanced Research with 6D image acquisition module for wildfield images and videos.

Data analysis

BD FACS Sortware for processing and analyzing FACS data.

Zeiss ZEN2 software package for processing Airyscan images.

CellProfiler 1.0.5122 and MATLAB R2017a for processing and analyzing lipid droplet distribution (Supplementary Fig 5e) and PMP22-mCardinal fluorescence (Supplementary Fig 5g) with custom software scripts available at https://bitbucket.org/szkabel/

lipidanalyser/get/master.zip.

Graphpad Prism 7.04 for graphs and statistical analysis.

All softwares above are available freely or commercially.

For manuscripts utilizing custom algorithms or software that are central to the research but not yet described in published literature, software must be made available to editors/reviewers. We strongly encourage code deposition in a community repository (e.g. GitHub). See the Nature Research guidelines for submitting code \& software for further information.

\section{Data}

Policy information about availability of data

All manuscripts must include a data availability statement. This statement should provide the following information, where applicable:

- Accession codes, unique identifiers, or web links for publicly available datasets

- A list of figures that have associated raw data

- A description of any restrictions on data availability

The data that support the findings of this study are available from the corresponding author upon reasonable request. 
Please select the one below that is the best fit for your research. If you are not sure, read the appropriate sections before making your selection. $\bigotimes$ Life sciences Behavioural \& social sciences Ecological, evolutionary \& environmental sciences

For a reference copy of the document with all sections, see nature.com/documents/nr-reporting-summary-flat.pdf

\section{Life sciences study design}

All studies must disclose on these points even when the disclosure is negative.

Sample size No statistical method was used to predetermine sample size. Multiple targets were used for the test. Typically 2 cell lines were used for each endogenous target. Experiments were repeated independently as least once. For FACS experiments, each data point was obtained from tens of thousands of cells.

Data exclusions No data were excluded from analysis.

Replication Replication was successful in all cases. Experiments were repeated independently at least once. Results with endogenous targets were consistent between different cell lines. Conclusions were consistent with all targets.

Randomization No randomization was performed.

Blinding Blinding did not apply as all experiments were done in cell culture and did not involve animal or human research participants.

\section{Reporting for specific materials, systems and methods}

We require information from authors about some types of materials, experimental systems and methods used in many studies. Here, indicate whether each material, system or method listed is relevant to your study. If you are not sure if a list item applies to your research, read the appropriate section before selecting a response.

Materials \& experimental systems

\begin{tabular}{l|l}
\hline$n / a$ & Involved in the study \\
$\square$ & $\bigotimes$ Antibodies \\
$\square$ & $\bigotimes$ Eukaryotic cell lines \\
$\square$ & $\square$ Palaeontology \\
$\bigotimes$ & $\square$ Animals and other organisms \\
$\bigotimes$ & $\square$ Clinical data
\end{tabular}

Methods

n/a Involved in the study

Х ChIP-seq

$\square \bigotimes$ Flow cytometry

Х $\square$ MRI-based neuroimaging

\section{Antibodies}

Antibodies used

Rabbit anti-GFP: Abcam ab290 (1:5,000)

Mouse anti-alpha Tubulin: Sigma B-5-1-2 (1:10,000)

Mouse anti-EGFR: DSHB CPTC-EGFR-1 $(0.5-1.0 \mu \mathrm{g} / \mathrm{ml})$

Mouse anti-PMP70: Sigma SAB4200181 (1:1,000)

Rabbit anti-seipin: in-house-generated antibody against C-terminal region of human seipin (1:400)

Mouse anti-HA: Sigma H9658 (1:5,000)

IRDye 800CW goat anti-mouse: Li-cor 926-32210 (1:10,000)

Alexa 680 goat anti-rabbit: Invitrogen A21109 (1:10,000).

Validation

Rabbit anti-GFP Abcam ab290 : tag antibody validated for WB; see manufacturer's website and validated in this study. Mouse anti-HA Sigma H9658: tag antibody validated for WB; see manufacturer's website and validated in this study.

Mouse anti-alpha Tubulin Sigma B-5-1-2: validated for WB in human samples, see manufacturer's website.

Mouse anti-EGFR DSHB CPTC-EGFR-1: validated for WB in human samples; see manufacturer's website and validated in this study.

Mouse anti-PMP70 Sigma SAB4200181: validated for WB in human samples, see manufacturer's website.

Rabbit anti-seipin in-house-generated antibody against C-terminal region of human seipin: validated for WB of overexpressed human seipin in this study.

IRDye $800 \mathrm{CW}$ goat anti-mouse and Alexa 680 goat anti-rabbit: secondary antibodies validated for WB by the see manufacturer's and in this study. 
Policy information about cell lines

Cell line source(s)

A431 cells (ATCC, Cat\#CRL-1555); A549 cells (ATCC, Cat\#CCL-185); HEK293A cells (Invitrogen, R70507).

Authentication

A431 cells, A549 cells and HEK293A cells were confirmed with visual inspection of the morphologies of the cells (compared to pictures provided by the vendors). A431 cells were also confirmed with unusually high level of EGFR expression through tagging with minilAA7-mEGFP tag (over 2-fold higher GFP level compared to LMNA in heterozygously tagged cell lines) and through western blot with anti-EGFR antibody. No further authentication was performed for the three cell lines.

Mycoplasma contamination

The cells were tested for mycoplasma contamination with PCR and the results were negative.

Commonly misidentified lines (See ICLAC register)

No commonly misidentified cell lines were used in this study.

\section{Flow Cytometry}

\section{Plots}

Confirm that:

Х The axis labels state the marker and fluorochrome used (e.g. CD4-FITC).

Х The axis scales are clearly visible. Include numbers along axes only for bottom left plot of group (a 'group' is an analysis of identical markers).

\All plots are contour plots with outliers or pseudocolor plots.

\A numerical value for number of cells or percentage (with statistics) is provided.

\section{Methodology}

Sample preparation

Single-cell suspensions were stored on ice prior to analysis. Cells were filtered through a $35 \mu \mathrm{m}$ nylon mesh before reading them in the Flow Cytometer.

Instrument

BD Influx cell sorter (BD Biosciences-US)

Software

BD FACS ${ }^{\text {TM }}$ Sortware

Cell population abundance

Single cloning to obtain homozygously tagged cell lines post sorting was successful.

Gating strategy

Cells were gated with SSC, FSC for cells, and trigger pulse width for singlets.

X Tick this box to confirm that a figure exemplifying the gating strategy is provided in the Supplementary Information. 